\title{
Stroke Mimics in the Acute Setting: Role of Multimodal CT Protocol
}

\author{
(D). Prodi, (D). Danieli, (DC. Manno, (D)A. Pagnamenta, (D). Pravatà, (D). Roccatagliata, (D). Städler, (D) C.W. Cereda, and \\ (iD) A. Cianfoni \\ O.
}

\begin{abstract}
BACKGROUND AND PURPOSE: Ischemic stroke can be mimicked by nonischemic conditions. Due to emphasis on the rapid treatment of acute ischemic stroke, it is crucial to identify these conditions to avoid unnecessary therapies and potential complications. We investigated the performance of the multimodal CT protocol (unenhanced brain CT, CTA, and CTP) to discriminate stroke mimics from acute ischemic stroke.
\end{abstract}

MATERIALS AND METHODS: We retrospectively selected multimodal CT studies performed for clinical suspicion of acute ischemic stroke in our center in a 24-month period, including patients with at least 1 follow-up imaging study (brain CT or MR imaging). Hemorrhagic strokes were excluded. We measured the performance of multimodal CT, comparing the original diagnostic results with the final clinical diagnosis at discharge.

RESULTS: Among 401 patients, a stroke mimic condition was diagnosed in 89 (22\%), including seizures (34.8\%), migraine with aura attack (12.4\%), conversion disorder (12.4\%), infection (7.9\%), brain tumor (7.9\%), acute metabolic condition (6.7\%), peripheral vertigo (5.6\%), syncope $(5.6 \%)$, transient global amnesia (3.4\%), subdural hematoma (1.1\%), cervical epidural hematoma (1.1\%), and dural AVF (1.1\%). Multimodal CT sensitivity, specificity, and accuracy were $24.7 \%, 99.7 \%$, and $83 \%$. Multimodal CT revealed peri-ictal changes in 13/31 seizures and diagnosed $7 / 7$ brain tumors, $1 / 1$ dural AVF, and 1/1 subdural hematoma. CT perfusion played a pivotal diagnostic role.

CONCLUSIONS: Multimodal CT demonstrated low sensitivity but high specificity in the diagnosis of stroke mimics in the acute setting. The high specificity of multimodal CT allows ruling out stroke and thereby avoiding unnecessary revascularization treatment in patients with diagnosis of a stroke mimic.

ABBREVIATIONS: $\mathrm{CECT}=$ contrast-enhanced $\mathrm{CT}$; EEG = electroencephalogram; iCVE $=$ ischemic cerebrovascular event; $\mathrm{SM}=$ stroke mimics; $T$ max $=$ time-to-maximum

$\mathbf{T}$ he clinical management of ischemic stroke requires a fast diagnostic workflow to refer patients to early revascularization. ${ }^{1}$ One of the challenges in the diagnosis of ischemic stroke is

Received June 22, 2021; accepted after revision October 6.

From the Departments of Neuroradiology (E.Prodi, L.D., E.Pravatà, A.C.) and Neurology (C.M., C.S., C.W.C.), Neurocenter of Southern Switzerland, EOC, Lugano, Switzerland; Unit of Clinical Epidemiology (A.P.), Ente Ospedaliero Cantonale, Bellinzona, Switzerland; Department of Intensive Care Medicine (A.P.), Ente Ospedaliero Cantonale, Mendrisio, Switzerland; Division of Pneumology (A.P.), University Hospital of Geneva, Geneva, Switzerland; Department of Health Science (DISSAL) (L.R.), University of Genova, Genova, Italy; Department of Neuroradiology (A.C.), Inselspital Bern, University of Bern, Bern, Switzerland; and Faculty of Biomedical Sciences (E. Pravatà), Università della Svizzera Italiana, Lugano, Switzerland.

Carlo W. Cereda and Alessandro Cianfoni contributed equally to this work. Please address correspondence to Elena Prodi, MD, Neurocenter of Southern Switzerland, Department of Neuroradiology, Via Tesserete 46, Lugano, 6900, Switzerland; e-mail: elena.prodi@eoc.ch

- Indicates open access to non-subscribers at www.ajnr.org

Indicates article with online supplemental data.

http://dx.doi.org/10.3174/ajnr.A7379 related to the presence of stroke mimic conditions, which are nonischemic disorders with clinical presentations similar to those of stroke. These disorders include seizures, systemic infections, brain tumors, toxic-metabolic conditions, positional vertigo, syncope, migraine, transient global amnesia, dementia, demyelinating disease, conversion disorder, and others. ${ }^{2,3}$ The prevalence of stroke mimics (SM) has been reported in up to $31 \%$ of patients with suspected acute stroke referred to the emergency department. ${ }^{4}$ Although complications of IV rtPA treatment in SM are rare, ${ }^{5,6}$ an accurate diagnosis is desirable to avoid unnecessary treatment and possible complications. Timely brain imaging is critical in the management of patients in this setting. MR imaging with diffusion-weighted imaging is the most sensitive technique to diagnose ischemic stroke. However, MR imaging is not routinely available in the emergency setting in many facilities; therefore, CT remains the first imaging method of choice in most centers. NCCT reliably rules out brain hemorrhage, but its 
sensitivity to identify early signs of brain ischemia is low in the first 6 hours from the onset of symptoms. ${ }^{7,8}$ Complementary CT techniques add further information on the presence, extent, and pathogenesis of ischemic stroke. CTA allows evaluating the status of neck and brain vessels and identifying a proximal arterial occlusion. CTP readily identifies areas of hypoperfused brain and allows estimating the presence of an ischemic core and an ischemic penumbra. ${ }^{9}$

A multimodal CT approach, including NCCT, CTA, and CTP, is used in many centers for the diagnosis of acute stroke, ${ }^{8}$ though the use of perfusion is not required by actual guidelines in the first 6 hours from stroke onset. ${ }^{1}$ Although multimodal CT has been reported to be able to distinguish some SM such as seizures, migraine, space-occupying lesions, infections, toxic-metabolic conditions, and others, ${ }^{10}$ there are scarce systematic studies on the diagnostic performance of multimodal CT in the diagnosis of SM, with most studies focusing on the diagnostic performance of CTP in seizures as a subgroup of SM. ${ }^{11-13}$

In this study, we aimed to evaluate the overall diagnostic performance of multimodal CT for the diagnosis of SM not limited to seizures in the setting of acute stroke care.

\section{MATERIALS AND METHODS}

This is a retrospective study based on a single medical center with a comprehensive stroke unit that offers 24/7 admission of patients with acute stroke, clinical and imaging diagnoses, and systemic and endovascular treatment.

The study was approved by the local institutional Ethics Committee, Comitato Etico Cantonale. The study cohort included all consecutive patients who underwent a multimodal CT examination in the acute setting for suspected acute stroke in a 24-month period. Exclusion criteria were as follows: hemorrhagic stroke; incomplete multimodal CT (ie, lack of $\geq 1$ imaging protocol component); examinations judged to be of nondiagnostic quality by the original interpreting neuroradiologist due to artifacts, poor patient cooperation, or other technical issues; and lack of at least 1 imaging follow-up (CT or MR imaging) necessary to confirm or rule out an acute brain ischemic lesion.

\section{Triage of Clinical Patients}

At our center, patients with clinical suspicion of acute ischemic stroke are promptly referred to a multimodal CT stroke imaging protocol after clinical evaluation by a neurologist, including an NIHSS evaluation.

\section{Multimodal CT at Admission}

Multimodal CT stroke imaging was performed using a dualsource 128-section CT scanner (Somatom Definition Flash; Siemens) as follows: NCCT of the head was followed by CTP acquired with a toggling-table technique, encompassing a brain coverage of $100 \mathrm{~mm}$ along the $\mathrm{z}$-axis for 45 seconds with a temporal resolution of 1.5 seconds. We then performed CTA in a dualenergy mode from the aortic arch to the vertex with a bolustracking technique, placing the density-monitoring ROI in the ascending aorta with a trigger threshold of $100 \mathrm{HU}$. Contrastenhanced CT (CECT) was acquired 4 minutes after the first injection of contrast medium when required. The details of CT technical parameters are reported in the Online Supplemental Data.

Postprocessing was performed by a CT technologist. Parametric qualitative maps of CBF, CBV, MTT, time-to-drain, and time-to-maximum (Tmax) were obtained from CTP data using a commercial perfusion package (VPCT-Neuro; Siemens). Arterial input and venous output time-attenuation curves were generated with ROIs automatically drawn on the anterior cerebral artery and the superior sagittal sinus, respectively. The CT technologist checked the correct positioning of the ROIs, the quality of the curves, and the presence of movement artifacts. Postprocessing of CTA source images was performed with multiplanar overlapping MIP reformatting. Images were sent, saved, and visualized in the PACS.

\section{Follow-up CT and MR Imaging Examinations}

Follow-up CT and MR imaging examinations were performed at a mean of 1.7 (SD, 2.6) days from admission before patient discharge, with MR imaging in $64 \%$ and CT in $36 \%$ of cases. NCCT was performed as described above. MR imaging was performed on a 3T scanner (Magnetom Skyra; Siemens) with a protocol consisting of at least the following sequences: axial DWI, axial FSE T2WI, axial FLAIR T2WI, and axial gradient recalled-echo $\mathrm{T} 2{ }^{\star} \mathrm{WI}$. The details of technical MR imaging parameters are shown in the Online Supplementary Data. Additional sequences, MRA, or postcontrast administration imaging were performed in selected cases as necessary.

\section{Imaging Diagnosis at Admission and Retrospective Imaging Diagnosis}

Images were rapidly interpreted and reported by a staff neuroradiologist who had access to the patient's clinical information. The group of 7 reporting neuroradiologists included physicians with a subspecialty in neuroradiology and experience ranging from 1 to 15 years. Radiology reports were archived in the radiology information system, integrated into the hospital informatics system.

For this study, 2 neuroradiologists (E.P. and L.D.) retrospectively reviewed, in consensus, the original reports of the multimodal CT study performed at admission and assigned the imaging diagnosis to 1 of 3 different categories: 1) evidence of acute ischemic vascular event (iCVE) on imaging; 2) evidence of an acute condition other that iCVE on imaging, likely the cause of the clinical presentation (SM); and 3) no evidence of acute iCVE or $\mathrm{SM}$ on imaging. The imaging diagnosis based on the report of the multimodal CT at admission was considered the index text to be evaluated for performance.

\section{Discharge Diagnosis and Retrospective Discharge Diagnosis}

The discharge diagnosis documented and archived in the hospital informatics system was considered the final diagnosis. The discharge report with the final diagnosis was signed in consensus by 2 staff stroke neurologists and 1 neurology fellow on the basis of relevant clinical, imaging, electroencephalogram (EEG), and laboratory data. For this study, the diagnosis at discharge was retrospectively reviewed in consensus by 1 neurology fellow (C.M.) and 1 neuroradiologist (E.P.) and dichotomized into 2 final 


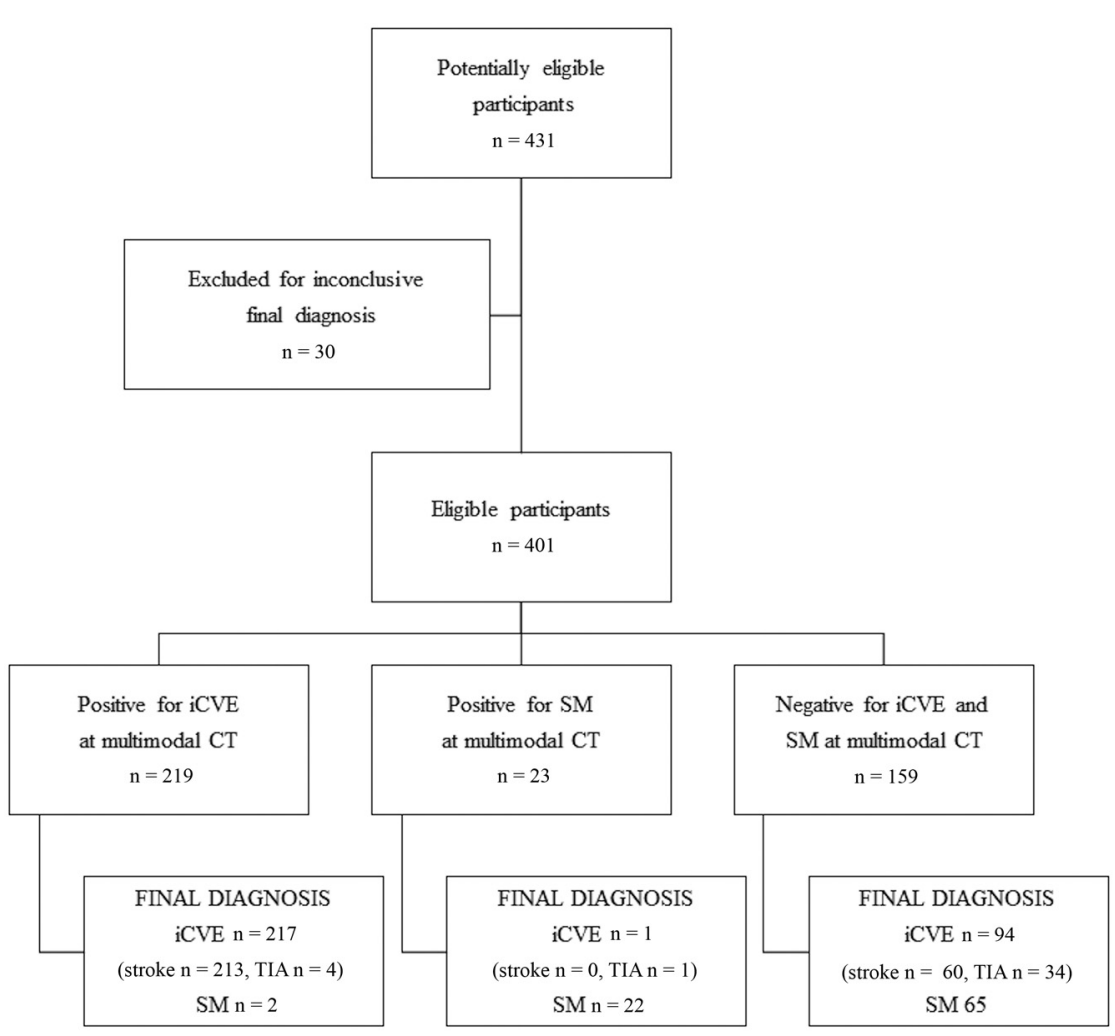

FIG 1. Study design diagram.

diagnoses: iCVE and SM. iCVE included stroke and TIA. ${ }^{14,15}$ For TIA, the likelihood of an ischemic event was rated with a version of the Precise Diagnostic Score scale. ${ }^{16}$ SM were cases in which the clinical details did not suggest an ischemic vascular etiology and another convincing explanation for the symptoms was found throughout supportive investigations. A few cases remained without a conclusive final diagnosis, even after evaluation of all available follow-up data. These included cases with less convincing clinical features, in which an alternative explanation for the clinical syndrome may have been present, but there was no definite proof of a nonstroke. These cases were excluded from the analysis. The final diagnosis at discharge for each patient was considered the reference diagnostic standard to establish true-positive, true-negative, false-positive, and false-negative and, therefore, to determine the accuracy of multimodal CT at admission.

\section{Data Analysis}

Descriptive statistics were presented as arithmetic mean with an SD or as median with interquartile range for quantitative data. Qualitative data were presented as absolute numbers with percentages. Comparisons of quantitative data among the 3 multimodal CT groups were performed with the Kruskal-Wallis test. With a statistically significant $P$ value, we performed post hoc tests (Mann-Whitney), taking into account the multiple comparisons. Qualitative data among groups of patients were compared using the $\chi^{2}$ test. With statistically significant results, we made post hoc comparisons with the appropriate critical level adjustment.
Comparisons between the diagnostic test and the reference standard permitted calculating sensitivity, specificity, positive and negative predictive values, positive and negative likelihood ratios, and accuracy with the corresponding 95\% confidence intervals. Contemplating an option of uncertainty in the multimodal CT results, we built $3 \times 2$ contingency tables. In $3 \times 2$ tables, we considered nonevaluable results as negative results, assuming a higher prevalence of a final diagnosis of iCVE. ${ }^{17}$

All tests were conducted 2-sided, and $P$ values $<.05$ were considered statistically significant. STATA, Version 15 (StatCorp) and MedCalc (MedCalc Software) were used for all statistical analyses.

\section{RESULTS}

Four hundred thirty-one patients fulfilled the inclusion criteria; 30 patients were excluded from the analysis due to an uncertain final diagnosis. Therefore 401 patients were included in our study population: 219 men and 182 women (mean age, 71 [SD 14] years; range, 25-97 years).

The results of multimodal CT at admission (index test) were categorized as diagnostic of iCVE $(n=219)$, diagnostic of SM $(n=23)$, and negative for both iCVE and SM $(n=159)$.

The clinical final diagnosis (reference standard) was dichotomized into diagnoses of iCVE $(n=312)$ and SM $(n=89)$. The iCVEs were further subdivided into diagnoses of stroke $(n=273)$ and TIA $(n=39)$ (Fig 1$)$.

The sex distribution was similar among different categories of multimodal CT diagnoses (male/female ratios, respectively, 122:97, 15:8, 82:77 for the categories of multimodal CT diagnoses of iCVE and SM, and negative for both iCVE and SM, $P=.298$ ) and final diagnoses (male/female ratios, respectively, 180:132 and 39:50 for the final diagnoses of iCVE and SM, $P=.087$ ).

Age distribution significantly differed between the categories of final diagnosis, respectively, 73 (SD, 13) years in the final diagnosis of iCVE and $66(\mathrm{SD}, 11)$ years in the final diagnosis of SM $(P=.002)$.

The prevalence of SM in our population was $22 \%$, including seizures $(n=31,34.8 \%)$, migraine with aura attack $(n=11,12.4 \%)$, conversion disorder $(n=11,12.4 \%)$, infection $(n=7,7.9 \%)$, brain tumor $(n=7,7.9 \%)$, acute metabolic condition $(n=6,6.7 \%)$, peripheral vertigo $(n=5,5.6 \%)$, syncope $(n=5,5.6 \%)$, transient global amnesia $(n=3,3.4 \%)$, subdural hematoma $(n=1,1.1 \%)$, cervical epidural hematoma $(n=1,1.1 \%)$ and dural AVF $(n=1$, $1.1 \%$ ) (Table 1 ). More specifically, infections included 1 viral undetermined encephalitis and 6 systemic infections; brain tumors were all primitive glial tumors; and metabolic causes included 2 cases of hepatic encephalopathy, 2 cases of drug-related encephalopathy, 1 case of hyponatremia, and 1 case of hypoglycemia. 
Statistical analysis results are shown in Table 2. Multimodal CT sensitivity, specificity, and accuracy for the diagnosis of SM were $24.7 \%$ (95\% CI, 16.2\%-35\%), 99.7\% (95\% CI, 98.2\%-100\%), and $83 \%$ (95\% CI, 79\%-86.6\%), respectively. Multimodal CT revealed peri-ictal changes in 13/31 seizures, 7/7 brain tumors, $1 /$ 1 dural AVF, and 1/1 subdural hematoma, while it revealed no relevant abnormal findings in 18/31 seizures, 11/11 migraines with aura attack, 11/11 conversion disorders, 7/7 infections, 5/5 cases of peripheral vertigo, 6/6 metabolic conditions, 5/5 cases of syncope, $3 / 3$ cases of transient global amnesia, and 1/1 cervical epidural hematoma. When we excluded a priori undetectable SM conditions on multimodal CT (conversion disorders, peripheral vertigo, syncope, transient global amnesia), multimodal CT sensitivity, specificity, and accuracy were $33.9 \%$ (95\% CI, $22.6 \%-$ 46.7\%), 99.7\% (95\% CI, 98.4\%-100\%), and 89\% (95\% CI, 85.6\%$91.9 \%)$, respectively.

The specificity of multimodal CT for the diagnosis of SM in our study was high: Only 2/85 SM were diagnosed as stroke, and only $1 / 327$ iCVEs was misdiagnosed as a stroke mimic on multimodal CT. More specifically, in 1 case, a subtle cortico-subcortical density was interpreted as a stroke, despite normal findings on perfusion maps, and the final diagnosis was aseptic meningoencephalitis, while 1 other false-positive diagnosis of stroke was derived from an erroneous interpretation of hypoperfusion, non-matching vascular territories such as a watershed hemodynamic hypoperfusion, which was, instead, a postseizure abnormality. The false-positive stroke mimic occurred, instead, in a patient with a vague perfusion asymmetry, which was interpreted as a possible postseizure abnormality, but the final diagnosis was a TIA. No imaging diagnosis of the cervical epidural hematoma was obtained on admission multimodal CT due to subtle and overlooked findings at CTA of the neck.

The role of each of the multimodal CT techniques in the diagnosis of a stroke mimic is shown in Table 3.

Table 1: SM and stroke mimics with diagnostic findings at multimodal CT

\begin{tabular}{lc}
\hline \multicolumn{1}{c}{ SM $(\boldsymbol{n}=89)$} & $\begin{array}{c}\text { SM with Diagnostic Findings at } \\
\text { Multimodal CT }\end{array}$ \\
\hline Seizures $(n=31)(34.8 \%)$ & $13 / 31(42 \%)$ \\
Migraine with aura attack $(n=11)(12.4 \%)$ & None \\
Conversion disorders $(n=11)(12.4 \%)$ & None \\
Infections $(n=7)(7.9 \%)$ & None \\
Brain tumors $(n=7)(7.9 \%)$ & $7 / 7(100 \%)$ \\
Metabolic causes $(n=6)(6.7 \%)$ & None \\
Peripheral vertigo $(n=5)(5.6 \%)$ & None \\
Syncope $(n=5)(5.6 \%)$ & None \\
Transient global amnesia $(n=3)(3.4 \%)$ & None \\
Subdural $(n=1)(1.1 \%)$ & $1 / 1(100 \%)$ \\
Dural arteriovenous fistula $(n=1)(1.1 \%)$ & $1 / 1(100 \%)$ \\
Cervical epidural hematoma $(n=1)(1.1 \%)$ & None \\
\hline
\end{tabular}

A hyperperfusion pattern (increased $\mathrm{CBV}$ and $\mathrm{CBF} \pm$ reduced $\mathrm{MTT} / \mathrm{Tm} \max )$ was observed in 7/13 seizures and 6/7 tumors (Fig 2 and Online Supplemental Data), while 6/13 seizures and 1/7 tumors showed a hypoperfusion pattern (increased MTT/ Tmax \pm reduced CBV and CBF) (Online Supplemental Data). While CT + CTA showed positive findings in $41 \%$ of detected SM, perfusion CT alone showed positive findings in $95.5 \%$ of them.

We also evaluated the sensitivity, specificity, and accuracy of multimodal

Table 2: Statistical analysis results

\begin{tabular}{|c|c|c|c|c|}
\hline & $\begin{array}{l}\text { Multimodal CT in the } \\
\text { Diagnosis of SM }\end{array}$ & $\begin{array}{c}\text { Multimodal CT in the Diagnosis } \\
\text { of SM (Excluding A } \\
\text { Priori CT, Undetectable SM) }\end{array}$ & $\begin{array}{l}\text { Multimodal CT in } \\
\text { the Diagnosis of } \\
\text { Definite Stroke (TIA } \\
\text { Excluded) }\end{array}$ & $\begin{array}{l}\text { Multimodal CT in } \\
\text { the Diagnosis of } \\
\text { iCVE (TIA Included) }\end{array}$ \\
\hline SE (\%) (95\% CI) (\%) & 24.7 (16.2-35) & $33.9(22.6-46.7)$ & 78 (72.6-82.8) & $69.6(64.1-74.6)$ \\
\hline SP (\%) (95\% CI) (\%) & $99.7(98.2-100)$ & 99.7 (98.4-100) & 97.8 (92.1-99.7) & 97.8 (92.1-99.7) \\
\hline PLR (\%) (95\% CI) (\%) & $77.1(10.5-564.3)$ & 113.7 (15.6-828.9) & 34.7 (8.8-136.9) & $31(7.9-122.1)$ \\
\hline NLR (\%) (95\% CI) (\%) & $0.8(0.7-0.9)$ & $0.7(0.6-0.8)$ & $0.2(0.2-0.3)$ & $0.3(0.3-0.4)$ \\
\hline PPV (\%) (95\% CI) (\%) & 95.7 (75-99.4) & 95.7 (75.1-99.4) & 99.1 (96.4-99.8) & $99.1(96.5-99.8)$ \\
\hline NPV (\%) (95\% CI) (\%) & $82.3(80.5 .-84)$ & $88.6(86.8-90.3)$ & $59.2(53.6-64.5)$ & $47.8(43.6-52.1)^{\prime}$ \\
\hline $\mathrm{ACC}(\%)(95 \% \mathrm{Cl})(\%)$ & $83(79-86.6)$ & $89(85.6-91.9)$ & $82.9(78.6-86.6)$ & 75.8 (71.3-79.9) \\
\hline
\end{tabular}

Note:-SE indicates sensitivity; SP, specificity; PLR, positive likelihood ratio; NLR, negative likelihood ratio; PPV, positive predictive value; NPV, negative predictive value; ACC, accuracy.

Table 3: Role of each multimodal CT technique (NCCT, CTA, CTP, CECT) in the diagnosis of SM

\begin{tabular}{|c|c|c|c|}
\hline SM Detected on Multimodal CT & NCCT/CECT & CTA & CTP \\
\hline Seizure, $7 / 13$ (54\%) & $\mathrm{Neg}^{\mathrm{a}} / \mathrm{no} \mathrm{CE}$ & Neg & $\uparrow \mathrm{CBV} \uparrow \mathrm{CBF} \downarrow \mathrm{MTT} / \mathrm{Tmax}$ \\
\hline Seizure, $6 / 13$ (46\%) & $\mathrm{Neg} / \mathrm{no} \mathrm{CE}$ & Neg & $\downarrow \mathrm{CBV} \downarrow \mathrm{CBF} \downarrow \mathrm{MTT} / \mathrm{Tmax}$ \\
\hline Brain tumor, $6 / 7$ (86\%) & Hypodensity, mass effect/CE in $3 / 6$ & Neg & $\uparrow \mathrm{CBV} \uparrow \mathrm{CBF} \downarrow \mathrm{MTT} / \mathrm{Tmax}$ \\
\hline Brain tumor, 1/7 (14\%) & Hypodensity, mass effect/no CE & Neg & $\downarrow \mathrm{CBV} \downarrow \mathrm{CBF} \downarrow \mathrm{MTT} / \mathrm{Tmax}$ \\
\hline Subdural hematoma, $1 / 1$ & Subdural collection/no CE & Vessel displacement & $\mathrm{Neg}$ \\
\hline AVF, $1 / 1$ & $\mathrm{Neg} /$ redundant vessels on CECT & Redundant vessels & $\uparrow \mathrm{CBV} \uparrow \mathrm{CBF} \downarrow \mathrm{MTT} / \mathrm{Tmax}$ \\
\hline
\end{tabular}

Note:-Neg indicates negative for acute findings; CE, contrast enhancement; $\uparrow$, increased; $\downarrow$, decreased.

${ }^{a}$ Although negative for acute finding, NCCT showed the following in 4 different patients: 2 subdural collections and 1 cavernoma, which were already known from previous examinations, and 1 chronic left parietal middle cerebral infarct. 


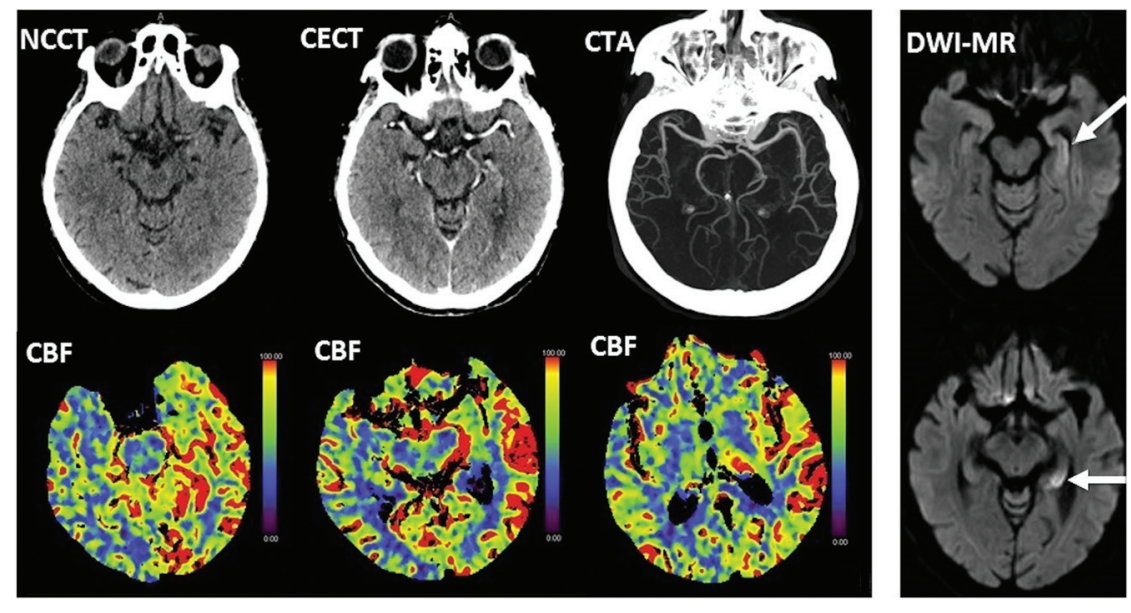

FIG 2. Left panel: elevated CBF consistent with hyperperfusion involving the left temporo-occipito-parietal cortex and left pulvinar in a 77-year-old woman with acute right-sided motor impairment, right hemineglect, aphasia, and conjugate eye deviation to the right. Note normal findings on NCCT, CECT, and CTA. Right panel: Diffusion MR imaging excludes ischemia and demonstrates seizure changes (arrows) in the left hippocampus.

CT for the diagnosis of stroke, which were, respectively, $78 \%$ (95\% CI, 72.6\%-82.8\%), 97.8\% (95\% CI, 92.1\%-99.7\%), and $82.9 \%$ (95\% CI, 78.6\%-86.6\%), in line with previous reports. ${ }^{18-20}$ The sensitivity, specificity, and accuracy of multimodal CT in the diagnosis of total iCVE (stroke and TIA) were, respectively, 69.6\% (95\% CI, 64.1\%-74.6\%), 97.8\% (95\% CI, 92.1\%-99.7\%), and $75.8 \%$ (95\% CI, 71.3\%-79.9\%) (Table 2).

Among 89 patients with SM in our cohort, $82 \%$ underwent MR imaging at follow-up. MR imaging determined the diagnosis of SM in only 5 patients, with no relevant findings on multimodal CT (7\% of patients with SM examined by MR imaging). Among these, there were patients with transient global amnesia $(n=3)$ and postictal hippocampal $(n=1)$ and cortical $(n=1)$ diffusion restriction. The remainder of the diagnoses of SM were established on the basis of other anamnestic, serologic, CSF, or EEG findings.

\section{DISCUSSION}

In this study, we aimed to examine the overall performance of multimodal CT for the diagnosis of SM in the context of clinical suspicion of acute ischemic stroke, demonstrating low sensitivity but high specificity with high positive predictive values and fairly high negative predictive values (respectively, 25.6\%, 99.7\%, $95.6 \%$, and $83.8 \%$ ). So far in the literature, the performance of CTP has been assessed mostly with regard to seizure-related SM, with a recent case-control study on a large seizure-related stroke mimic population demonstrating sensitivity, specificity, and positive and negative predictive values, respectively, of $38 \%, 86 \%$, $42 \%$, and $83 \%$ for CTP hyperperfusion in patients with ongoing seizure (ictal state). ${ }^{11}$

In our population, as in previous studies, ${ }^{6,21}$ seizure was the most common stroke mimic. We were able to recognize 13/31 (42\%) seizures on the basis of perfusion abnormalities on multimodal CT, with a hyperperfusion pattern in 7/13 (54\%) and a hypoperfusion pattern in $6 / 13(46 \%)$. Because in many of our patients, EEG was not obtained at the time of multimodal CT acquisition, it is not possible to correlate these perfusion abnormality patterns with an ictal or postictal state.

Van Cauwenberge et $\mathrm{al}^{11}$ reported that CTP can reliably differentiate acute ischemic stroke from ictal seizure, respectively, characterized by corticosubcortical hypoperfusion and cortical hyperperfusion. Conversely, they showed that CTP findings may overlap in acute ischemic stroke and postictal seizure, both characterized by corticosubcortical hypoperfusion, though seizure-related hypoperfusion typically follows a nonvascular distribution. In our study, the specificity of multimodal CT for the diagnosis of SM was high: Only 2/85 SM were diagnosed as stroke, and only 1/327 iCVEs was misdiagnosed as a stroke mimic; 2 of 3 of these misdiagnoses were related to a misinterpretation of hypoperfusion findings, with a postictal seizure erroneously interpreted as a stroke and vice versa. Therefore, our results confirm the potential overlap of CTP findings between postictal hypoperfusion and acute ischemic stroke.

We detected all brain tumors present in our cohort by multimodal CT. Abnormal findings were visible on NCCT and CECT due to the presence of mass effect, vasogenic edema, and/or contrast enhancement. CTP showed hyperperfusion in 6/7 (86\%) and hypoperfusion in 1/7 (14\%) brain tumors. Perfusion abnormalities in brain tumors may be related to the nature of the lesion, with typical hyperperfusion in high-grade gliomas, or to induced seizure. ${ }^{22}$

In our cohort, none of the patients with an attack of migraine with aura had recognizable multimodal CT findings, though perfusion abnormalities have been described in migraine, mostly on MR imaging studies demonstrating abnormalities ranging from hypoperfusion to hyperperfusion, with a typical nonvascular distribution, ${ }^{23-25}$ which can be associated to an increase of distal cortical vessel representation. ${ }^{26}$

None of the toxic-metabolic conditions or infections in our cohort were detected by multimodal CT, though in these cases, NCCT may variably show areas of hypoattenuation or swelling, with possible contrast enhancement. Perfusion findings have also been anecdotally described in case reports such as in Wernicke encephalopathy, ${ }^{27}$ Hashimoto encephalopathy, ${ }^{28}$ and viral infections. ${ }^{29,30}$

In addition, we did not encounter, in our cohort, luxury perfusion conditions, which may pose a challenge in a differential diagnosis between a postreperfusion iCVE and a stroke mimic. Luxury perfusion represents, in fact, the recovery of CBF to normal or increased levels in regions of infarcted brain when re-vascularization is established after a stroke, sometimes spontaneously. Luxury perfusion commonly occurs in subacute strokes but has also been reported in $<24$-hour strokes. ${ }^{31}$ 
In our study, the sensitivity of multimodal CT for the diagnosis of SM was low (25.6\%) and remained low (34.4\%; 95\% CI, 22.9-47.3), even excluding SM, which are a priori considered to be undetectable by multimodal CT (such as conversion disorder, positional vertigo, syncope, and transient global amnesia). Due to its low sensitivity, multimodal CT cannot, therefore, be considered an efficient diagnostic tool for SM. In our cohort, 82\% of SM underwent MR imaging at follow-up, and MR imaging has determined the diagnosis of SM in only 5 patients, with no relevant findings on multimodal CT (7\% of SM examined by MR imaging). Although MR imaging allows ruling out stroke and may give a further hint of the diagnosis of SM, the diagnosis of these conditions relies on other complementary tests, such as serum and CSF analysis or EEG findings.

Conversely, our study outlines the high specificity of multimodal CT for the diagnosis of SM. This may support the clinician in the choice of avoiding revascularization treatment in SM with positive findings on multimodal CT. Among the different multimodal CT techniques, CTP was the most useful in the detection of SM, demonstrating abnormal findings in $95.5 \%$ of the overall diagnoses of SM performed by multimodal CT. Despite the current debate about the usefulness of CTP in patients with stroke in the first 6 hours from symptom onset, ${ }^{1}$ CTP has the potential to detect perfusion abnormalities related to stroke mimic conditions and in cases in which brain CT is typically silent, such as in seizures.

Limitations in this study are related to the retrospective type of the analysis and to the relatively small number of subjects, which might have precluded some uncommon SM from being part of our population. There are, indeed, other vascular dysregulation conditions that might have a clinical presentation similar to that of stroke and may show CTP abnormalities, that we did not encounter in this study, such as posterior reversible encephalopathy syndrome ${ }^{32}$ and reversible cerebral vasoconstriction syndrome. ${ }^{33}$

Another limitation is related to a selection bias in our study population, because we included only patients who underwent a full multimodal CT protocol at admission. We, therefore, excluded either patients with iCVE or those with SM without a multimodal CT protocol (ie, patients transferred from neighboring hospitals where CTP was not performed in the acute setting). Also, patients with a very high clinical suspicion of SM (such as those with hypoglycemia, positional vertigo, and migraine) might not have been referred to the multimodal CT protocol. Nonetheless, the prevalence of SM in our cohort was $21 \%$, within the range of previous reports. ${ }^{4,34}$ Subjects with a final diagnosis of SM were younger than those with a final diagnosis of iCVE, and this difference was statistically significant. This finding is in line with the expected epidemiology of patients with SM. Indeed, clinical indicators of a mimic condition, though nonspecific, include young age and lack of a history of hypertension, hyperlipidemia, atrial fibrillation, diabetes, and coronary artery disease. ${ }^{21}$ Moreover, some patients with minor stroke who presented at admission as having a TIA, with complete symptom regression, did not undergo a full multimodal CT protocol and were, therefore, excluded from the study.

Finally, this was a monocentric study, and it may limit the generalizability of our findings. In particular, our series benefitted from consultant neurology review before imaging and a subspecialist neuroradiology review at time of imaging.

\section{CONCLUSIONS}

Multimodal CT has a low sensitivity for the diagnosis of SM. MR imaging and further investigation (serum and CSF analysis and EEG) are required to obtain a definite diagnosis of these conditions. On the contrary, the high specificity of multimodal CT for the diagnosis of stroke mimic can support the clinician in the choice of avoiding revascularization treatment in patients diagnosed with SM on multimodal CT.

\section{ACKNOWLEDGMENTS}

We thank Matteo Merli, Istituto Imaging della Svizzera Italiana, Ente Ospedaliero Cantonale, Lugano, Switzerland, for CT technical support; and the Clinical Trial Unit, Ente Ospedaliero Cantonale, Bellinzona, Switzerland, for assistance in research project development.

Disclosure forms provided by the authors are available with the full text and PDF of this article at www.ajnr.org.

\section{REFERENCES}

1. Powers WJ, Rabinstein AA, Ackerson T, et al. Guidelines for the Early Management of Patients with Acute Ischemic Stroke: 2019 Update to the 2018 Guidelines for the Early Management of Acute Ischemic Stroke-a Guideline for Healthcare Professionals from the American Heart Association/American Stroke Association. Stroke 2019;50:e344-418 CrossRef Medline

2. Norris JW, Hachinski VC. Misdiagnosis of stroke. The Lancet 1982;319:328-31 CrossRef

3. Libman RB, Wirkowski E, Alvir J, et al. Conditions that mimic stroke in the emergency department implications for acute stroke trials. Arch Neurol 1995;52:1119-22 CrossRef Medline

4. Merino JG, Luby M, Benson RT, et al. Predictors of acute stroke mimics in 8187 patients referred to a stroke service. J Stroke Cerebrovasc Dis 2013;22:e397-403 CrossRef Medline

5. Tsivgoulis G, Zand R, Katsanos AH, et al. Safety of intravenous thrombolysis in stroke mimics. Stroke 2015;46:1281-87 CrossRef Medline

6. Winkler DT, Fluri F, Fuhr P, et al. Thrombolysis in stroke mimics frequency, clinical characteristics, and outcome. Stroke 2009;40:1522-25 CrossRef Medline

7. Lev MH, Farkas J, Rodriguez VR, et al. CT angiography in the rapid triage of patients with hyperacute stroke to intraarterial thrombolysis: accuracy in the detection of large vessel thrombus. J Comput Assist Tomogr 2001;25:520-28 Medline

8. Wintermark M, Sanelli PC, Albers GW, et al; Society of Neurointerventional Surgery. Imaging recommendations for acute stroke and transient ischemic attack patients: a joint statement by the American Society of Neuroradiology, the American College of Radiology and the Society of Neurointerventional Surgery. J Am Coll Radiol 2013;10:828-32 CrossRef Medline

9. Demeestere J, Wouters A, Christensen S, et al. Review of perfusion imaging in acute ischemic stroke. Stroke 2020;51:1017-24 CrossRef Medline

10. Vilela P. Acute stroke differential diagnosis: stroke mimics. Eur J Radiol 2017;96:133-44 CrossRef Medline

11. Van Cauwenberge MG, Dekeyzer S, Nikoubashman O, et al. Can perfusion CT unmask postictal stroke mimics? A case-control study of 133 patients. Neurology 2018;91:E1918-27 CrossRef Medline 
12. Lucas L, Gariel F, Menegon P, et al. Acute ischemic stroke or epileptic seizure? Yield of CT perfusion in a code stroke situation. AJNR Am J Neuroradiol 2021;42:49-56 CrossRef Medline

13. Gonzalez-Martinez A, Trillo S, de Quirós C, et al. Predictors of perfusion computed tomography alterations in stroke mimics attended as stroke code. Eur J Neurol 2021;28:1939-48 CrossRef Medline

14. Sacco RL, Kasner SE, Broderick JP, et al; Council on Nutrition, Physical Activity and Metabolism. An updated definition of stroke for the 21st century: a statement for healthcare professionals from the American Heart Association/American Stroke Association. Stroke 2013;44:2064-89 CrossRef Medline

15. Easton JD, Saver JL, Albers GW, et al; Interdisciplinary Council on Peripheral Vascular Disease. Definition and evaluation of transient ischemic attack: a scientific statement for healthcare professionals from the American Heart association/American Stroke Association Stroke Council; Council on Cardiovascular Surgery and Anesthesia; Council on Cardiovascular Radiology and Intervention; Council on Cardiovascular Nursing-and the Interdisciplinary Council on Peripheral Vascular Disease. Stroke 2009;40:2276-93 CrossRef Medline

16. Cereda CW, George PM, Inoue M, et al. Inter-rater agreement analysis of the Precise Diagnostic Score for suspected transient ischemic attack. Int J Stroke 2016;11:85-92 CrossRef Medline

17. Schuetz GM, Schlattmann P, Dewey M. Use of $\mathbf{3} \times \mathbf{2}$ tables with an intention to diagnose approach to assess clinical performance of diagnostic tests: meta-analytical evaluation of coronary CT angiography studies. BMJ 2012;345:e6717 CrossRef Medline

18. Kloska SP, Nabavi DG, Gaus C, et al. Acute stroke assessment with CT: do we need multimodal evaluation? Radiology 2004;233:79-86 CrossRef Medline

19. Biesbroek JM, Niesten JM, Dankbaar JW, et al. Diagnostic accuracy of CT perfusion imaging for detecting acute ischemic stroke: a systematic review and meta-analysis. Cerebrovasc Dis 2013;35:493-501 CrossRef Medline

20. Shen J, Li X, Li Y, et al. Comparative accuracy of CT perfusion in diagnosing acute ischemic stroke: a systematic review of 27 trials. PLoS One 2017;12:e0176622 CrossRef Medline

21. Hand PJ, Kwan J, Lindley RI, et al. Distinguishing between stroke and mimic at the bedside: the Brain Attack Study. Stroke 2006;37:769-75 CrossRef Medline
22. Jain R. Perfusion CT imaging of brain tumors: an overview. AJNR Am J Neuroradiol 2011;32:1570-77 CrossRef Medline

23. Floery D, Vosko MR, Fellner FA, et al. Acute-onset migrainous aura mimicking acute stroke: MR perfusion imaging features. AJNR Am J Neuroradiol 2012;33:1546-52 CrossRef Medline

24. Förster A, Wenz H, Kerl HU, et al. Perfusion patterns in migraine with aura. Cephalalgia 2014;34:870-76 CrossRef Medline

25. Jadhav V. Computed tomography perfusion abnormalities in migraine mimicking acute stroke presentation (P06.157.). Neurology 2013;80(7):P06.157

26. Masuzaki M, Utsunomiya $H$, Yasumoto $S$, et al. A case of hemiplegic migraine in childhood: transient unilateral hyperperfusion revealed by perfusion $\mathrm{MR}$ imaging and MR angiography. AJNR Am J Neuroradiol 2001;22:1795-97 Medline

27. Bhan A, Advani R, Kurz KD, et al. Wernicke's encephalopathy mimicking acute onset stroke diagnosed by CT perfusion. Case Rep Neurol Med 2014;2014:673230 CrossRef Medline

28. Cho $\mathrm{BH}$, Cheon $\mathrm{K}$, Lee $\mathrm{KY}$. Hashimoto encephalopathy mimicking acute ischemic stroke: perfusion-weighted magnetic resonance imaging. J Clin Neurol 2018;14:586-87 CrossRef Medline

29. de Lucas EM, Mandly GA, Gutiérrez A, et al. Computed tomography perfusion usefulness in early imaging diagnosis of herpes simplex virus encephalitis. Acta Radiol 2006;47:878-81 CrossRef Medline

30. Vallabhaneni D, Naveed MA, Mangla R, et al. Perfusion imaging in autoimmune encephalitis. Case Rep Radiol 2018;2018:3538645 CrossRef Medline

31. Nagar VA, McKinney AM, Karagulle AT, et al. Reperfusion phenomenon masking acute and subacute infarcts at dynamic perfusion CT: confirmation by fusion of CT and diffusion-weighted MR images. AJR AM J Roentgenol 2009;193:1629-38 CrossRef Medline

32. Hedna VS, Stead LG, Bidari S, et al. Posterior reversible encephalopathy syndrome (PRES) and CT perfusion changes. Int J Emerg Med 2012;5:12 CrossRef Medline

33. Miller TR, Shivashankar R, Mossa-Basha M, et al. Reversible cerebral vasoconstriction syndrome, Part 2: diagnostic work-up, imaging evaluation, and differential diagnosis. AJNR Am J Neuroradiol 2015;36:1580-88 CrossRef Medline

34. Hemmen TM, Meyer BC, McClean TL, et al. Identification of nonischemic stroke mimics among 411 code strokes at the University of California, San Diego, Stroke Center. J Stroke Cerebrovasc Dis 2008;17:23-25 CrossRef Medline 